\title{
Reactive Sulfur Species: Kinetics and Mechanism of the Oxidation of Cystine by Hypochlorous Acid to Give $\mathbf{N}, \mathbf{N}$ '-Dichlorocystine
}

\author{
Péter Nagy and Michael T. Ashby* \\ Department of Chemistry and Biochemistry, University of Oklahoma, 620 \\ Parrington Oval, Norman, OK 73019
}

Submitted March 19, 2005; E-mail: mashby@ou.edu

\section{SUPPORTING INFORMATION}

Figure S1. Typical trace at $290 \mathrm{~nm}\left(\lambda_{\max }\right.$ for $\left.\mathrm{OCl}^{-}\right)$reflecting the disappearance of $\mathrm{OCl}^{-}$ for the reaction of $1.6 \mathrm{mM}$ cystine, $2.5 \mathrm{mM} \mathrm{OCl}^{-}$, at $\mathrm{pH}=11.3$ ( $0.1 \mathrm{M}$ phosphate) and $\mathrm{I}=$ $0.45 \mathrm{M}\left(\mathrm{NaCl}+\mathrm{Na}_{2} \mathrm{HPO}_{4}+\mathrm{Na}_{3} \mathrm{PO}_{4}\right)$ at $\mathrm{T}=293 \mathrm{~K}$ fit to a second order equation (red) and residual.

Figure S2. Typical trace at $260 \mathrm{~nm}\left(\lambda_{\max }\right.$ for NDC) reflecting the appearance of NDC for the reaction of $1.6 \mathrm{mM}$ cystine, $2.5 \mathrm{mM} \mathrm{OCl}^{-}$, at $\mathrm{pH}=11.3(0.1 \mathrm{M}$ phosphate) and $\mathrm{I}=$ $0.45 \mathrm{M}\left(\mathrm{NaCl}+\mathrm{Na}_{2} \mathrm{HPO}_{4}+\mathrm{Na}_{3} \mathrm{PO}_{4}\right)$ at $\mathrm{T}=293 \mathrm{~K}$ fit to a second order equation (red) and residual.

Figure S3. Time-resolved ${ }^{1} \mathrm{H}$ NMR spectra illustrating a transient spectrum that is assigned to NCC: $[\mathrm{HOCl}]_{0}^{\mathrm{T}}=5.0 \mathrm{mM}$, [cystine $]_{0}=2.5 \mathrm{mM}, \mathrm{T}=293 \mathrm{~K}$. The sample was prepared at $\mathrm{pH} 7.5$ (225 $\mathrm{mM}$ phosphate). 
Table S1. Effect of [Cystine] on the Observed Rate.

Table S2. Effect of $\left[\mathrm{OH}^{-}\right]$on the Observed Rate.

Table S3. Effect of $\mathrm{pH}$ on the Observed Rate. 


\section{Derivation of Rate Law}

Determine $[\mathrm{HOCl}]$ in terms of $[\mathrm{HOCl}]^{\mathrm{T}}=[\mathrm{HOCl}]+\left[\mathrm{OCl}^{-}\right]$:

$$
[\mathrm{HOCl}]=\frac{\left[\mathrm{H}^{+}\right][\mathrm{HOCl}]^{\mathrm{T}}}{\left[\mathrm{H}^{+}\right]+\mathrm{K}_{1}}
$$

Describe $\left[\right.$ cystine $\left.^{1-}\right]$ and $\left[\right.$ cystine $\left.{ }^{2-}\right]$ in terms of $\left[\right.$ cystine $\left.^{0}\right]$ :

$$
\begin{gathered}
\text { cystine } \left.^{1-}\right]=\frac{\left.\mathrm{K}_{2} \text { cystine }^{0}\right]}{\left[\mathrm{H}^{+}\right]} \\
{\left[\text {cystine }^{2-}\right]=\frac{\mathrm{K}_{2} \mathrm{~K}_{3}\left[\text { cystine }^{0}\right]}{\left[\mathrm{H}^{+}\right]^{2}}}
\end{gathered}
$$

Solve for $\left[\right.$ cystine $\left.{ }^{0}\right]$ in terms of $[\text { cystine }]_{0}^{\mathrm{T}}$ :

$$
\left[\text { cystine }^{0}\right]=\frac{\left[\mathrm{H}^{+}\right]^{2}[\text { cystine }]_{0}^{\mathrm{T}}}{\left[\mathrm{H}^{+}\right]^{2}+\mathrm{K}_{2}\left[\mathrm{H}^{+}\right]+\mathrm{K}_{2} \mathrm{~K}_{3}}
$$

Solve for $\left[\right.$ cystine $\left.^{1-}\right]$ in terms of $[\text { cystine }]_{0}^{\mathrm{T}}$ :

$$
\left[\text { cystine }^{1-}\right]=\frac{\mathrm{K}_{2}\left[\mathrm{H}^{+}\right][\text {cystine }]_{0}^{\mathrm{T}}}{\left[\mathrm{H}^{+}\right]^{2}+\mathrm{K}_{2}\left[\mathrm{H}^{+}\right]+\mathrm{K}_{2} \mathrm{~K}_{3}}
$$

Solve for $\left[\right.$ cystine $\left.{ }^{2-}\right]$ in terms of $[\text { cystine }]_{0}^{\mathrm{T}}$ :

$$
\left[\text { cystine }^{2-}\right]=\frac{\mathrm{K}_{2} \mathrm{~K}_{3}[\text { cystine }]_{0}^{\mathrm{T}}}{\left[\mathrm{H}^{+}\right]^{2}+\mathrm{K}_{2}\left[\mathrm{H}^{+}\right]+\mathrm{K}_{2} \mathrm{~K}_{3}}
$$

The Rate Law:

$$
\frac{d[\mathrm{NCC}]}{d \mathrm{t}}=\left(\mathrm{k}_{4} \frac{\mathrm{K}_{2}\left[\mathrm{H}^{+}\right][\text {cystine }]_{0}^{\mathrm{T}}}{\left[\mathrm{H}^{+}\right]^{2}+\mathrm{K}_{2}\left[\mathrm{H}^{+}\right]+\mathrm{K}_{2} \mathrm{~K}_{3}}+\mathrm{k}_{5} \frac{\mathrm{K}_{2} \mathrm{~K}_{3}[\text { cystine }]_{0}^{\mathrm{T}}}{\left[\mathrm{H}^{+}\right]^{2}+\mathrm{K}_{2}\left[\mathrm{H}^{+}\right]+\mathrm{K}_{2} \mathrm{~K}_{3}}\right) \frac{\left[\mathrm{H}^{+}\right][\mathrm{HOCl}]^{\mathrm{T}}}{\left[\mathrm{H}^{+}\right]+\mathrm{K}_{1}}
$$

Simplify the Rate Law:

$$
\frac{d[\mathrm{NCC}]}{d \mathrm{t}}=\frac{\mathrm{K}_{2}\left(\mathrm{k}_{4}\left[\mathrm{H}^{+}\right]^{2}+\mathrm{K}_{3} \mathrm{k}_{5}\left[\mathrm{H}^{+}\right]\right)[\text {cystine }]_{0}^{\mathrm{T}}}{\left(\mathrm{K}_{1}+\left[\mathrm{H}^{+}\right]\right)\left(\left[\mathrm{H}^{+}\right]^{2}+\mathrm{K}_{2}\left[\mathrm{H}^{+}\right]+\mathrm{K}_{2} \mathrm{~K}_{3}\right)}[\mathrm{HOCl}]^{\mathrm{T}}
$$




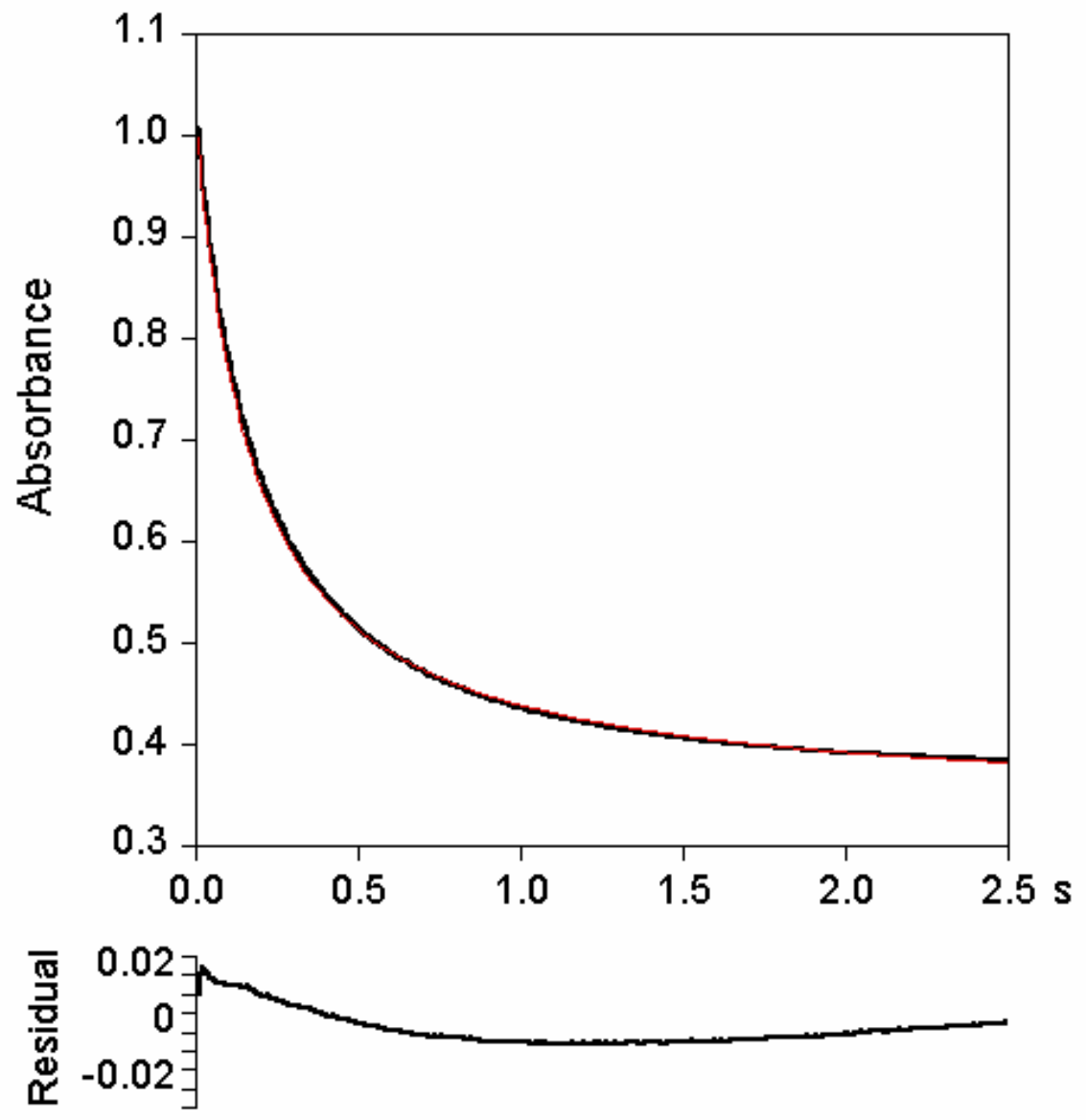

Figure S1. Typical trace at $290 \mathrm{~nm}\left(\lambda_{\max }\right.$ for $\left.\mathrm{OCl}^{-}\right)$reflecting the disappearance of $\mathrm{OCl}^{-}$for the reaction of $1.6 \mathrm{mM}$ cystine, 3.2 $\mathrm{mM} \mathrm{OCl}^{-}$, at $\mathrm{pH}=11.3(0.1 \mathrm{M}$ phosphate $)$ and $\mathrm{I}=0.45 \mathrm{M}(\mathrm{NaCl}$ $\left.+\mathrm{Na}_{2} \mathrm{HPO}_{4}+\mathrm{Na}_{3} \mathrm{PO}_{4}\right)$ at $\mathrm{T}=293 \mathrm{~K}$ fit to a second order equation (red) and residual. 


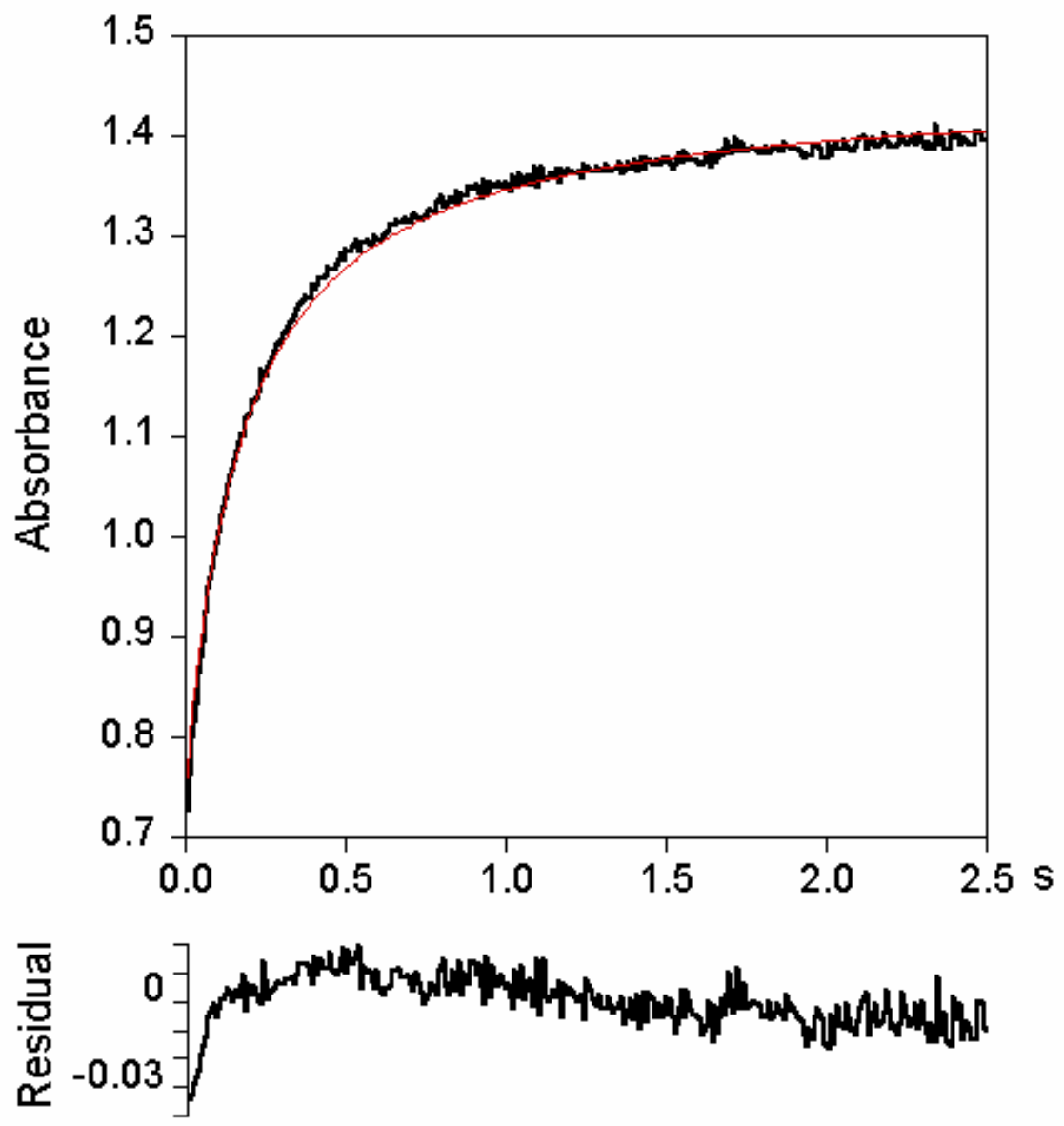

Figure S2. Typical trace at $260 \mathrm{~nm}\left(\lambda_{\max }\right.$ for NDC) reflecting the appearance of NDC for the reaction of $1.6 \mathrm{mM}$ cystine, 3.2 $\mathrm{mM} \mathrm{OCl}$, at $\mathrm{pH}=11.3(0.1 \mathrm{M}$ phosphate $)$ and $\mathrm{I}=0.45 \mathrm{M}(\mathrm{NaCl}$ $\left.+\mathrm{Na}_{2} \mathrm{HPO}_{4}+\mathrm{Na}_{3} \mathrm{PO}_{4}\right)$ at $\mathrm{T}=293 \mathrm{~K}$ fit to a second order equation (red) and residual. 


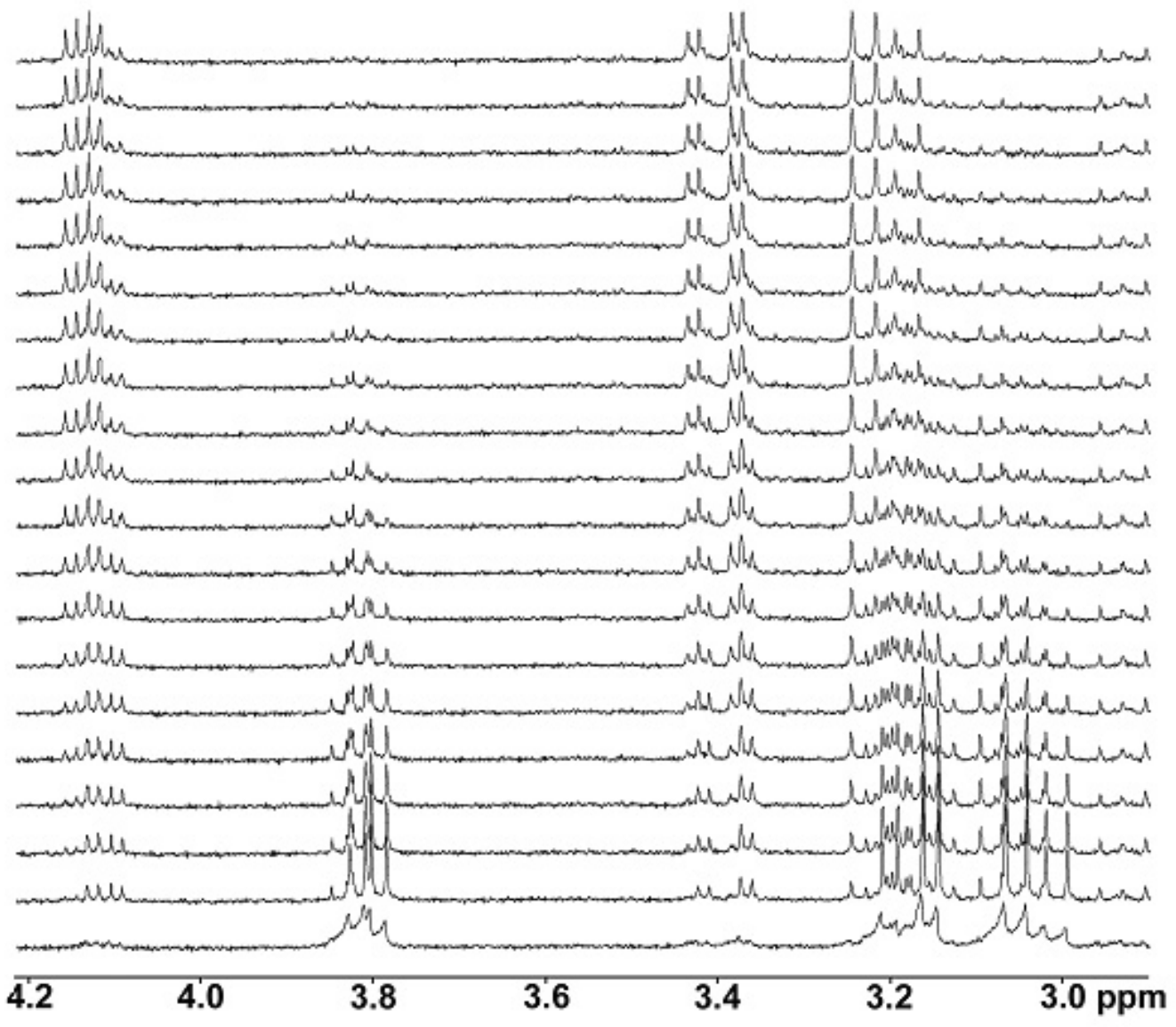

Figure S3. Time-resolved ${ }^{1} \mathrm{H}$ NMR spectra illustrating the transient spectrum that is assigned to $\mathrm{NCC}:[\mathrm{HOCl}]_{0}^{\mathrm{T}}=5.0 \mathrm{mM},[\text { cystine }]_{0}=2.5 \mathrm{mM}$, $\mathrm{T}=293 \mathrm{~K}$. The sample was prepared at $\mathrm{pH} 7.5$ (225 mM phosphate). From bottom to top, the first spectrum is at $\mathrm{T}=3 \mathrm{~min} 17 \mathrm{sec}$ and the delay between spectra was $2 \min 10 \mathrm{sec}$. 
Table S1. Effect of [Cystine] on the Observed Rate. ${ }^{a}$

$\begin{array}{ccc}\text { [Cystine] }(\mathrm{M}) & \text { av. } \mathrm{k}_{\mathrm{obs}}\left(\mathrm{s}^{-1}\right) & \text { std. dev. } \\ 1.50 \times 10^{-3} & 3.58 & 7.42 \times 10^{-2} \\ 3.00 \times 10^{-3} & 6.13 & 1.18 \times 10^{-1} \\ 4.50 \times 10^{-3} & 9.95 & 1.19 \times 10^{-1} \\ 6.00 \times 10^{-3} & 1.38 \times 10^{1} & 1.78 \times 10^{-1} \\ 7.50 \times 10^{-3} & 1.85 \times 10^{1} & 3.60 \times 10^{-1} \\ 9.00 \times 10^{-3} & 2.22 \times 10^{1} & 1.70 \times 10^{-1} \\ 1.20 \times 10^{-2} & 3.08 \times 10^{1} & 1.16 \\ 1.50 \times 10^{-2} & 3.78 \times 10^{1} & 8.45 \times 10^{-1}\end{array}$

${ }^{\mathrm{a}}\left[\mathrm{OCl}^{-}\right]=0.15 \mathrm{mM}, \mathrm{pH}=11.3(0.1 \mathrm{M}$ phosphate $)$, ionic strength: 1 $\mathrm{M}\left(\mathrm{NaClO}_{4}+\mathrm{Na}_{2} \mathrm{HPO}_{4}+\mathrm{Na}_{3} \mathrm{PO}_{4}+\mathrm{Na}_{2}\right.$ (cystine)), $\mathrm{T}=293 \mathrm{~K}$. Kinetic traces were evaluated at $300 \mathrm{~nm}$. 
Table S2. Effect of $\left[\mathrm{OH}^{-}\right]$on the Observed Rate. ${ }^{\mathrm{a}}$

$\begin{array}{ccc}1 /[\mathrm{OH}]\left(\mathrm{M}^{-1}\right) & \text { av. } \mathrm{k}_{\mathrm{obs}}\left(\mathrm{s}^{-1}\right) & \text { std. dev. } \\ 3.09 \times 10^{2} & 3.58 & 7.42 \times 10^{-2} \\ 6.46 \times 10^{2} & 8.25 & 1.08 \times 10^{-1} \\ 1.29 \times 10^{3} & 1.66 \times 10^{1} & 3.30 \times 10^{-1} \\ 3.09 \times 10^{3} & 4.18 \times 10^{1} & 8.94 \times 10^{-1} \\ 8.32 \times 10^{3} & 1.13 \times 10^{2} & 3.17 \\ 3.24 \times 10^{4} & 3.62 \times 10^{2} & 4.30 \times 10^{1}\end{array}$

${ }^{\mathrm{a}}\left[\mathrm{OCl}^{-}\right]=0.15 \mathrm{mM}$, [cystine] $=1.5 \mathrm{mM}, \mathrm{pH}=11.3(0.1 \mathrm{M}$ phosphate), ionic strength: $1 \mathrm{M}\left(\mathrm{NaClO}_{4}+\mathrm{Na}_{2} \mathrm{HPO}_{4}+\mathrm{Na}_{3} \mathrm{PO}_{4}+\right.$ $\mathrm{Na}_{2}$ (cystine)), $\mathrm{T}=293 \mathrm{~K}$. Kinetic traces were evaluated at $300 \mathrm{~nm}$. 
Table S3. Effect of $\mathrm{pH}$ on the Observed Rate. ${ }^{\mathrm{a}}$

$\begin{array}{ccc}\mathrm{pH} & \text { av. } \text { keff }\left(\mathrm{M}^{-1} \mathrm{~s}^{-1}\right) & \text { std. dev } \\ 6.59 & 8.68 \times 10^{4} & 2.01 \times 10^{3} \\ 6.80 & 1.33 \times 10^{5} & 6.52 \times 10^{3} \\ 6.89 & 1.44 \times 10^{5} & 3.45 \times 10^{3} \\ 7.01 & 1.55 \times 10^{5} & 4.17 \times 10^{4} \\ 7.07 & 2.00 \times 10^{5} & 8.43 \times 10^{3} \\ 7.20 & 2.19 \times 10^{5} & 1.77 \times 10^{4} \\ 7.28 & 2.56 \times 10^{5} & 1.17 \times 10^{4} \\ 7.34 & 3.55 \times 10^{5} & 6.23 \times 10^{4} \\ 7.57 & 4.20 \times 10^{5} & 4.71 \times 10^{3} \\ 7.73 & 5.64 \times 10^{5} & 3.65 \times 10^{3} \\ 7.87 & 5.76 \times 10^{5} & 2.59 \times 10^{4} \\ 8.07 & 5.12 \times 10^{5} & 7.00 \times 10^{4} \\ 8.11 & 5.72 \times 10^{5} & 2.59 \times 10^{4} \\ 8.34 & 4.53 \times 10^{5} & 2.36 \times 10^{3} \\ 8.49 & 4.20 \times 10^{5} & 8.82 \times 10^{3} \\ 8.75 & 4.25 \times 10^{5} & 4.91 \times 10^{4} \\ 9.02 & 3.10 \times 10^{5} & 4.71 \times 10^{3} \\ 9.31 & 1.92 \times 10^{5} & 1.50 \times 10^{3} \\ 9.33 & 2.12 \times 10^{5} & 1.77 \times 10^{3} \\ 9.76 & 1.07 \times 10^{5} & 2.36 \times 10^{3} \\ 10.30 & 4.43 \times 10^{4} & 2.89 \times 10^{2} \\ 10.42 & 3.90 \times 10^{4} & 4.01 \times 10^{2} \\ 10.91 & 1.20 \times 10^{4} & 1.18 \times 10^{2} \\ & & \end{array}$

${ }^{\mathrm{a}}\left[\mathrm{OCl}^{-}\right]=0.06 \mathrm{mM}$, [cystine $]=0.6 \mathrm{mM}, \mathrm{pH}=6.59-10.91(0.1 \mathrm{M}$ phosphate), ionic strength: $1 \mathrm{M}\left(\mathrm{NaClO}_{4}+\mathrm{NaH}_{2} \mathrm{PO}_{4}+\mathrm{Na}_{2} \mathrm{HPO}_{4}+\right.$ $\mathrm{Na}_{3} \mathrm{PO}_{4}+\mathrm{Na}_{2}$ (cystine)), $\mathrm{T}=278 \mathrm{~K}$. Kinetic traces were evaluated at 255 (from $\mathrm{pH}=6.59-8.11)$ or $300 \mathrm{~nm}($ from $\mathrm{pH}=8.34-10.91)$. 